\title{
SWI Susceptibility Vessel Sign in Patients Undergoing Mechanical Thrombectomy for Acute Ischemic Stroke
}

(D) N.F. Belachew, (D). Dobrocky, (DE.B. Aleman, (D)T.R. Meinel, (D) A. Hakim, (D). Vynckier, (D) M. Arnold, (DD.J. Seiffge, (D) R. Wiest, (D) P. Mordasini, (D). Gralla, (D). Fischer, (DE.I. Piechowiak, and (D). Kaesmacher

\begin{abstract}
BACKGROUND AND PURPOSE: The frequency and clinical significance of the susceptibility vessel sign in patients with acute ischemic stroke remains unclear. We aimed to assess its prevalence in patients with acute ischemic stroke undergoing mechanical thrombectomy and to analyze its association with interventional and clinical outcome parameters in that group.
\end{abstract}

\begin{abstract}
MATERIALS AND METHODS: Six hundred seventy-six patients with acute ischemic stroke and admission MR imaging were reviewed retrospectively. Of those, 577 met the eligibility criteria for further analysis. Imaging was performed using a 1.5T or 3T MR imaging scanner. Associations between baseline variables, interventional and clinical outcome parameters, and susceptibility vessel sign were determined with multivariable logistic regression models. Results are shown as adjusted ORs with $95 \%$ Cls.

RESULTS: The susceptibility vessel sign was present in $87.5 \%(n=505)$ of patients and associated with tandem occlusion (adjusted OR, 3.3; 95\% CI, 1.1-10.0; $P=.032$ ) as well as successful reperfusion, defined as an expanded TICl score of $\geq 2 \mathrm{~b}$ (adjusted OR, 2.4; $95 \% \mathrm{Cl}, 1.28-4.6 ; P=.007)$. The susceptibility vessel sign was independently associated with functional independence (mRS $\leq$ 2: adjusted OR, 2.1; 95\% Cl, 1.1-4.0; $P=.028$ ) and lower mortality (adjusted OR, 0.4; 95\% Cl, 0.2-0.7; $P=.003$ ) at 90 days, even after adjusting for successful reperfusion. The susceptibility vessel sign did not influence the number of passes performed during mechanical thrombectomy, the first-pass reperfusion, or the risk of peri- or postinterventional complications.

CONCLUSIONS: The susceptibility vessel sign is an MR imaging phenomenon frequently observed in patients with acute ischemic stroke and is associated with successful reperfusion after mechanical thrombectomy. However, superior clinical functional outcome and lower mortality noted in patients showing the susceptibility vessel sign could not be entirely attributed to higher reperfusion rates.
\end{abstract}

ABBREVIATIONS: AIS = acute ischemic stroke; aOR = adjusted OR; MT = mechanical thrombectomy; SVS = susceptibility vessel sign

$\mathbf{S}_{\mathrm{cos}}^{\mathrm{w}}$ WI is an MR imaging sequence particularly sensitive to compounds that distort the local magnetic field and therefore allow the detection of very small amounts of blood products and calcium. Due to the paramagnetic property of deoxygenated hemoglobin in trapped blood cells, it can also be used to locate thrombus material in occluded vessels after acute

Received February 3, 2021; accepted after revision May 27.

From the Departments of Diagnostic and Interventional Neuroradiology (N.F.B., T.D., E.B.A., A.H., R.W., P.M., J.G., E.I.P., J.K.), Neurology (T.R.M., J.V., M.A., D.J.S., U.F.), and Diagnostic, Interventional and Pediatric Radiology (J.K.), Inselspital, Bern University Hospital, and University of Bern, Bern, Switzerland.

E.I. Piechowiak and J. Kaesmacher have shared last author position.

Please address correspondence to Nebiyat Filate Belachew, MD, Department of Diagnostic and Interventional Neuroradiology, Inselspital, Freiburgstr 18, CH-3010, Switzerland; e-mail: nebiyatfilate.belachew@insel.ch; @NFBelachew

- Indicates open access to non-subscribers at www.ajnr.org

Indicates article with online supplemental data.

http://dx.doi.org/10.3174/ajnr.A7281 ischemic stroke (AIS), which may be seen as a distinct loss of signal within the affected vessel. ${ }^{1}$ While this phenomenon was first described as the "gradient recalled echo susceptibility vessel sign (GRE SVS)" in T2*-weighted imaging, ${ }^{2,3}$ the clot-detection rate has proved to be significantly higher with SWI, which provides better spatial resolution and is therefore superior in visualizing blood-degradation products. ${ }^{4}$ However, not all thromboembolic vessel occlusions are visible on SWI. Their detectability depends on the composition of the clot, making erythrocyte-rich thrombi more likely to result in an occlusion that is apparent on SWI. ${ }^{5,6}$ While mechanical thrombectomy (MT) has proved effective in treating large-vessel occlusion in patients with AIS, ${ }^{7}$ data on the prevalence of the SVS in these patients are inconsistent. ${ }^{2-4,8-12}$ Although thrombus composition is known to influence the success of $\mathrm{MT},{ }^{13-17}$ it is unclear whether the SVS is associated with successful reperfusion and good clinical outcome. , $9-12,18,19$ Our aim was to assess the 


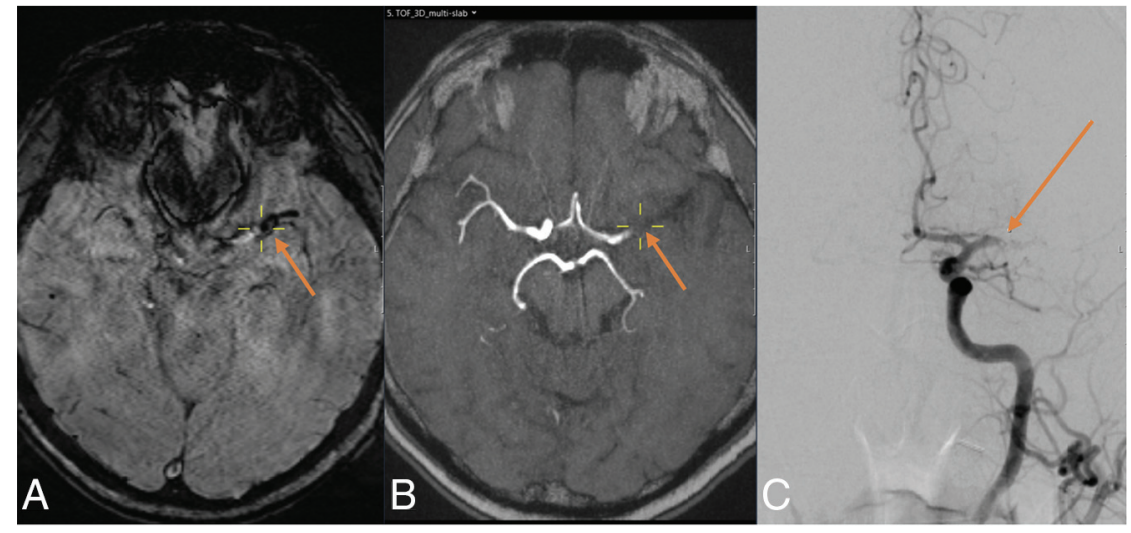

FIG 1. A 70-year-old male patient with acute ischemic stroke. The SVS is visible on SWI $(A)$ as a distinct, circumscribed signal loss along the main trunk of the left MCA, representing the occlusive thrombus. Complete occlusion of the left MCA main trunk (M1 segment) is also seen on the arterial TOF sequence $(B)$ and on DSA $(C)$. Yellow crosshairs are centered on the proximal end of the vessel occlusion on SWI $(A)$ and arterial TOF $(B)$. The arrow points to the proximal end of the vessel occlusion on SWI, arterial TOF and DSA.

prevalence of the SVS in patients with AIS undergoing MT and to analyze its association with interventional and clinical outcome parameters.

\section{MATERIALS AND METHODS Inclusion Criteria}

Clinical and radiologic data were gathered from the records of patients with AIS who underwent MT at our hospital between January 2010 and December 2018. The inclusion criteria were as follows: 1) a final clinical diagnosis of AIS; 2) SWI performed on admission; 3) symptomatic occlusion of at least one intracranial artery on angiography; and 4) the patient having undergone endovascular treatment in the form of MT. SWI quality was classified as "excellent" (if there were no artifacts), "good" (if there were minor artifacts), "poor" (if there were major artifacts, but the SVS was assessable), or "very poor" (if the SVS was not assessable due to major artifacts). The SVS was considered "technically undeterminable" if the thrombus was masked due to its proximity to the skull base or being overlaid with other pathologies (eg, hemorrhage). Patients with very poor-quality SWI or a technically undeterminable SVS status were excluded.

Most patients with stroke admitted to our institution are scanned using MR imaging. However, the final decision on whether to perform MR imaging or CT is made by the neuroradiologists and neurologists in charge on a case-by-case basis depending on clinical aspects and contraindications. SWI was an inherent part of our stroke MR imaging protocol throughout this study, except when the neuroradiologists were confident that it would yield inconclusive results on the basis of the sequences performed beforehand (ie, due to the presence of foreign objects or motion artifacts). This study was approved by the local ethics committee.

\section{Analysis of Clinical Information}

Demographics, baseline characteristics, and clinical data such as age, sex, history of stroke, medication before AIS (antiplatelet treatments, anticoagulants, or statins), and cardiovascular risk factors such as hypertension, diabetes mellitus, dyslipidemia, and smoking habits were collected. In addition, we recorded the systolic and diastolic blood pressures on admission, the glucose levels on admission, the NIHSS score on admission, and stroke subtypes according to the Trial of Org 10172 in Acute Stroke Treatment (TOAST) classification. $^{20}$ Also, IV thrombolysis before imaging (transfer patients) and before MT, time from symptom onset/ last seen well to admission, time from symptom onset/last seen well to IV thrombolysis, time from symptom onset/last seen well to MT, and time from groin puncture to reperfusion were registered.

\section{Technical Information on MR Imaging}

Imaging was performed on a $1.5 \mathrm{~T}$ or $3 \mathrm{~T} \mathrm{MR}$ imaging scanner (1.5T: Magnetom Avanto or Magnetom Aera; 3T: Magnetom Verio; Siemens). Magnetom Avanto 1.5T SWI and 1.5T Magnetom Aera SWI were performed with the following parameters: TR, $49 \mathrm{~ms}$; TE, $40 \mathrm{~ms}$; flip angle, $15.0^{\circ}$; section thickness, 1.6, 1.8 , or $2.0 \mathrm{~mm}$; and intersection gap, $0 \mathrm{~mm}$. Magnetom Verio 3T SWI was performed with the following parameters: TR, $27 \mathrm{~ms}$; TE, $20 \mathrm{~ms}$; flip angle, $15.0^{\circ}$; section thickness, $2.0 \mathrm{~mm}$; and intersection gap, $0 \mathrm{~mm}$.

\section{Imaging Analysis}

The presence of SVS was evaluated retrospectively by 2 independent neuroradiologists (N.F.B. and E.B.A.) with 5 and 4 years of experience, respectively. Except for knowing which side was symptomatic, the raters were blinded to all clinical information and outcome parameters, and they were not involved in any patient treatment. SWI was classified as SVS + if a distinct signal loss corresponding to an occluded and symptomatic intracranial artery could be identified (Fig 1). Applying the definition used by Kang et al, ${ }^{9}$ we determined the SVS to be present even if its diameter was the same as or smaller than the diameter of the contralateral artery. However, the SVS was only classified as SVS+ if no alternative explanations for the signal loss were observed (ie, neighboring vein, petechial hemorrhage, or microcalcification in the neighboring parenchyma). SWIs in which the SVS was not present though a symptomatic vessel occlusion was apparent were categorized as SVS- (Fig 2). MR imaging field strength and time from symptom onset/last seen well to imaging were documented for each case. Additionally, the DWI-ASPECTS was evaluated.

\section{DSA and MT}

The primary site of occlusion was determined on conventional angiography. Tandem occlusions were also noted. However, only intracranial occlusions were considered for SVS evaluation. MT was performed by skilled interventional neuroradiologists 


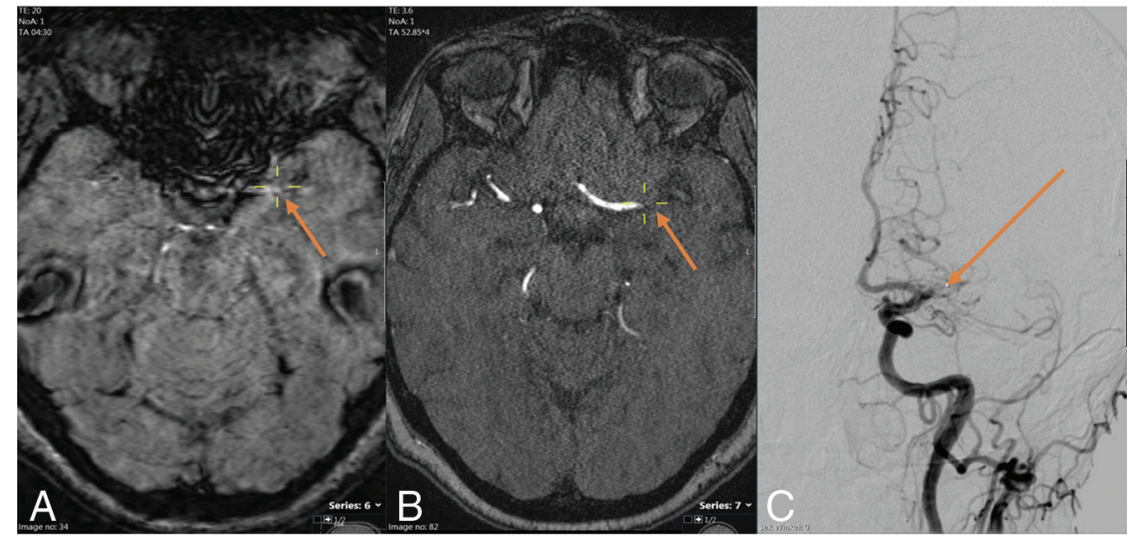

FIG 2. A 48-year-old female patient with acute ischemic stroke. SWI shows no SVS (A) Complete proximal occlusion of the left MCA main trunk ( $\mathrm{Ml}$ segment) is seen on the arterial TOF sequence $(B)$ and on DSA (C). Yellow crosshairs are centered on the proximal end of the vessel occlusion on SWI $(A)$ and arterial TOF $(B)$. The arrow points to the proximal end of the vessel occlusion on SWI, arterial TOF, and DSA.

according to the current clinical practice guidelines and institutional protocols. The expanded TICI score ${ }^{21}$ was documented after the first pass and at the end of MT. Also, the total number of passes performed during MT was recorded. First-pass reperfusion and overall reperfusion were deemed successful if the expanded TICI was $2 \mathrm{~b}$ or better. DSA was screened for embolization into previously unaffected (ie, new) territories and for peri-interventional complications (vasospasm, dissection, and perforation) by a research fellow with 3 years of experience.

\section{Outcome}

The clinical outcome was assessed by evaluating the NIHSS 24 hours after treatment as well as the mRS and mortality 90 days after treatment. The NIHSS was evaluated by the attending neurologist, whereas the mRS was evaluated by a neurologist or a study nurse, in person or by a telephone call. Early neurologic recovery was defined as a decrease in the NIHSS score 24 hours after treatment compared with admission, whereas functional independence was defined as mRS $\leq$ 2. Symptomatic intracranial hemorrhage within 48 hours after MT was assessed according to the European Cooperative Acute Stroke Study (ECASS II). ${ }^{22}$

\section{Statistical Analysis}

Data analysis was performed using the SPSS Software (Version 25.0; IBM). Continuous variables were compared using the MannWhitney $U$ test, whereas categoric variables were compared using the $\chi^{2}$ test. Multivariable binary logistic regression analyses were performed to determine the association between baseline parameters, tandem occlusion, and successful reperfusion, as well as functional independence and mortality at 90 days with the SVS. Adjustment was performed for all cofactors with $P<.15$ (sex, diabetes mellitus, prestroke $\mathrm{mRS}>2$, antiplatelet therapy, diastolic blood pressure, admission glucose, admission NIHSS, primary site of occlusion, tandem occlusion, and DWI-ASPECTS) as well as for additional cofactors that are known or suspected to influence the variables of interest (ie, age, sex, bridging therapy, stroke subtype, and symptomatic intracranial hemorrhage). Sensitivity analyses excluding patients with prestroke mRS $>2$ were performed for functional independence and mortality at 90 days. Interrater reliability was determined by calculating the Cohen $\kappa$. Results with 2 -tailed $P$ values $<.05$ were considered statistically significant and are shown as median comparisons with respective $P$ values or as an adjusted OR (aOR) with 95\% CIs.

\section{RESULTS}

From January 2010 to December 2018, one thousand three hundred seventeen patients underwent MT for AIS at our hospital. In 676 patients, an MR imaging was acquired on admission, and SWI was available for 614 of them. SVS status was assessable in $93.4 \%$ patients $(n=577 ; 37$ were excluded due to very poor-quality SWI or technically undeterminable SVS status) of whom $87.5 \%(n=505 / 577)$ were categorized as SVS + . An overview of the patient-selection process can be found in the Online Supplemental Data. SVS prevalence tended to be higher among patients who had received IV thrombolysis before MR imaging, but this difference was not statistically significant $(87.5 \%$ versus $78.6 \% ; P=.076$ ). Patients for whom the SVS was assessable did not differ significantly from patients with an unassessable SVS with regard to demographics and key outcome parameters (Online Supplemental Data). However, AIS patients with an admission CT instead of an admission MR imaging had significantly higher admission NIHSS scores, higher NIHSS scores at 24 hours, lower reperfusion rates, lower rates of functional independence at 90 days, and higher mortality rates at 90 days (Online Supplemental Data). Interrater reliability for SVS classification was strong $(\kappa=0.873, P<.001)$. The results for both the SVSand the SVS+ group are listed in the Online Supplemental Data.

The SVS- group had a higher percentage of female patients (62.5\% versus $49.7 \%$; $P=.042$ ), more patients diagnosed with diabetes mellitus before stroke $(27.8 \%$ versus $12.7 \% ; P=.001)$, more patients with prestroke dependency (mRS $>2: 16.7 \%$ versus $7.1 \% ; P=.005$ ), and higher DWI-ASPECTS (8 versus 8 ; $P=.006)$. Vessel occlusions in the anterior circulation tended to be seen more often in the ICA and proximal MCA (M1) for the SVS+ group and more often in the distal MCA (M2 and M3) for the SVS- group $(\mathrm{ICA}=8.3 \%$ versus $16.4 \%$; $\mathrm{M} 1=50.0 \%$ versus $53.1 \%$; $\mathrm{M} 2=25.0 \%$ versus $21.4 \%$; $\mathrm{M} 3=0.6 \%$ versus $1.4 \%$; $P=.013)$. By contrast, the SVS+ group had more tandem occlusions $(19.2 \%$ versus $5.6 \% ; P=.004)$. None of the other baseline characteristics differed between the 2 groups.

In a multivariable logistic regression model, diabetes mellitus before stroke (aOR, 0.431; 95\% CI, 0.204-0.912; $P=.028$ ) and prestroke dependency (mRS $>2$ : aOR, 0.390; 95\% CI, 0.174$0.875 ; P=.022)$ were associated with SVS- after adjusting for all factors with $P<.15$ (Online Supplemental Data). Although the SVS- group had a higher percentage of female patients, the same 


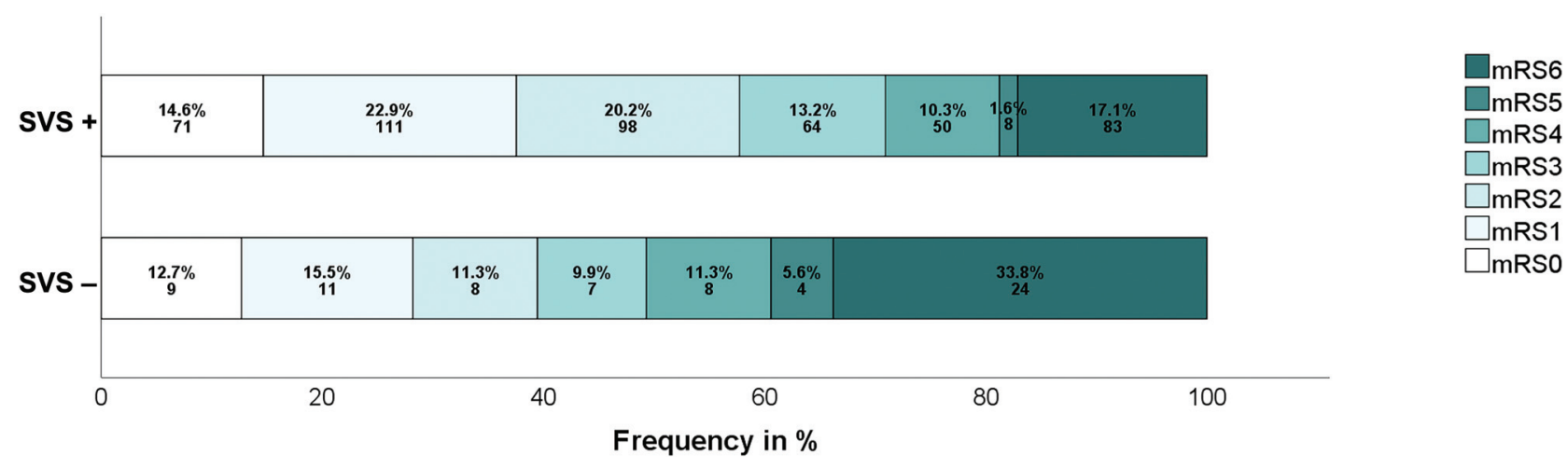

FIG 3. Distribution of the mRS according to SVS status. Data are expressed as percentages and total values. Prestroke mRS $>2$ for SVS + versus SVS-: $7.1 \%$ versus $16.7 \%(P=.005)$. Functional independence (mRS $\leq 2)$ for SVS + versus SVS- at 90 days: $59.7 \%$ versus $40.6 \%(P=.004)$.

model showed no association between sex and SVS status (aOR, 0.680 ; 95\% CI, $0.373-1.239 ; P=.208)$.

In a second model in which we compared patients on the basis of the presence of tandem pathology and adjusted for all covariates with $P<.15$ (Online Supplemental Data), tandem occlusions were associated with SVS+ intracranial vessel occlusions (aOR, 3.328; 95\% CI, 1.112-9.965; $P=.032$ ).

\section{Association between the SVS and Reperfusion}

Patients with SVS+ intracranial vessel occlusions had a higher rate of successful reperfusion after MT (84.6\% versus $72.2 \%$; $P=.009)$. Notably, the presence of the SVS had no influence on the number of passes performed during MT (1 versus $1 ; P=.552$ ), the first-pass reperfusion (58.6\% versus $52.9 \% ; P=.382$ ), or the likelihood of peri- or postinterventional complications (symptomatic intracranial hemorrhage: $4.2 \%$ versus $5.6 \%, P=0591$; embolization into previously unaffected [ie, new] territories: $4.2 \%$ versus $1.4 \%, P=.250$; peri-interventional complications: $14.3 \%$ versus $16.7 \%, P=.592)$. In a third logistic regression model adjusted for all covariates with $P<.15$ (Online Supplemental Data) as well as for age, bridging therapy, and stroke subtype, SVS + was associated with successful reperfusion after MT (expanded TICI score $\geq 2 \mathrm{~b}$ : aOR, 2.864; 95\% CI, 1.442-5.691; $P=.003$ ). This observation did not change regardless of whose rating (rater 1 or 2) was used for analysis (Online Supplemental Data).

\section{Association between the SVS and Clinical Outcome}

The SVS+ group showed superior early recovery 24 hours after treatment (NIHSS improvement: -4 versus $-2 ; P=.001)$ and a better clinical outcome 90 days after treatment (mRS $\leq 2: 55.4 \%$ versus $38.9 \% ; P=.004)$. Mortality was higher in the SVS- group (33.3\% versus $16.4 \% ; P=.001)$. Figure 3 shows the mRS distribution according to SVS status. In two separate multivariable regression models adjusted for the effects of all cofactors with $P<.15$ (Online Supplemental Data) as well as clinical predictors of good outcome (age, stroke subtype, bridging therapy, successful reperfusion, and symptomatic ICH), SVS+ was associated with a lower mRS score (mRS $\leq 2$ : aOR, 2.062; 95\% CI, $1.034-$ $4.115 ; P=.040$ ) and lower mortality (aOR, 0.351; 95\% CI, $0.167-$ $0.738 ; P=.006) 90$ days after treatment. These observations did not change regardless of the whose rating (rater 1 or 2) was used for analysis (Online Supplemental Data). A sensitivity analysis that excluded patients with prestroke mRS $>2$ and was adjusted for the first-line retrieval technique did not change these findings either (Online Supplemental Data).

\section{DISCUSSION}

The main findings of this study are the following: The SVS was present in $87.5 \%(n=505)$ of patients treated with MT for intracranial vessel occlusion and was associated with tandem occlusion as well as successful reperfusion. It was independently associated with functional independence and lower mortality rates 90 days after treatment, even after adjusting for successful reperfusion and showed no association with the number of passes performed during MT, the first-pass reperfusion, or the peri- and postinterventional complication rate.

While earlier studies included only a small number of patients, ${ }^{2,3,8,9,18}$ more recent ones have examined the SVS in larger study populations. ${ }^{10-12}$ However, because several important questions remain, larger studies are required. ${ }^{12}$

Early publications reported an overall SVS prevalence of $\sim 50 \%{ }^{2,3,8}$ in patients with AIS, whereas more recent studies have indicated that the prevalence is around $70 \%{ }^{9-12,18}$ Our findings suggest that the prevalence may be even higher. There are several possible explanations for these differences: 1) Sample size among the studies examining SVS varied widely, and small test populations may be prone to statistical inaccuracies that limit validity. ${ }^{11,12,23}$ 2) The eligibility criteria for MR imaging may differ between institutions and hospitals, leading to selection bias. For instance, whereas some hospitals scan patients regardless of special monitoring, others prefer CT when such monitoring is required. However, the SVS distribution may be different in patients whose condition is critical. 3) We only included patients who had undergone MT; consequently, patients who showed spontaneous reperfusion or had sufficient reperfusion after IV thrombolysis were excluded. Despite some evidence to the contrary, ${ }^{2,3}$ most studies suggested that SVS + intracranial vessel occlusions (particularly in the ICA and MCA) are less amenable to IV thrombolysis. ${ }^{8,24,25}$ Therefore, it is possible that the overall prevalence is lower if MR imaging is always performed before IV thrombolysis. 4) The comparability of SVS studies is often limited 
owing to differing inclusion criteria (eg, ICA/M1 occlusions only $^{8}$ versus other occlusion patterns ${ }^{3}$ ). 5) Not all studies adopted the same definition of SVS. Whereas some defined it as a signal loss within the margins of an acutely occluded vessel, ${ }^{9}$ others considered the SVS to be present only if the diameter of the signal loss exceeded that of the contralateral vessel. ${ }^{3}$ 6) The sensitivity for SVS may differ depending on the sequence ( $\mathrm{T} 2^{*}$ gradient recalled-echo versus $\mathrm{SWI}^{4}$ ) and the particular MR imaging scanner used (ie, its field strength and manufacturer). The slightly longer acquisition time for SWI compared with $\mathrm{T} 2^{\star}$ gradient recalled-echo sequences might be justified by the higher spatial resolution of SWI, which provides better visualization of blooddegradation products in distal or small thrombi and has proved to increase SVS sensitivity. ${ }^{4}$ 7) Indications for MT and access to MR imaging have evolved considerably since the SVS was first reported in 2000. ${ }^{1}$ Thus, study populations may differ significantly between current and earlier studies.

MT is more effective with erythrocyte-rich than with fibrinrich thrombi. ${ }^{14,26,27}$ Because erythrocyte-rich clots are more likely to result in an SVS+ intracranial vessel occlusion, ${ }^{5}$ SVS may also predict successful reperfusion after MT. If so, it could function as a noninvasive surrogate marker for thrombus composition. Although many studies have addressed this question, only one found an association between the SVS status and reperfusion success after MT. ${ }^{9-12,18}$ Other than the present study, Darcourt et $\mathrm{al}^{11,17}$ were the only investigators to report a significant relationship between SVS+ intracranial vessel occlusions and successful reperfusion after MT. This report raises the question of why earlier studies found no association. Some of the previously mentioned factors (ie, differing SVS definitions and variations in the sensitivity of SVS detection) may play a role. In addition, Bourcier et $\mathrm{al}^{10}$ have suggested that first-line MT techniques may affect reperfusion success in AIS patients with SVS. This hypothesis is currently being investigated prospectively (adaptatiVe Endovascular Strategy to the CloT MRI in Large Intracranial Vessel Occlusion [VECTOR] trial; ClinicalTrials.gov Identifier: NCT04139486). Future studies could also investigate whether certain stent-based retrieval techniques are superior to others in terms of reperfusion and/or periand postinterventional complication rates. Although our observations suggest that SVS + thrombi are easier to retrieve via MT than SVS- clots, most data so far suggest that they are less amenable to intravenous thrombolysis. Future studies may examine the risks and benefits of bridging therapy in patients with AIS, depending on the SVS status.

Few data are available on the association of SVS status with clinical outcome after MT. Bourcier et $\mathrm{al}^{18}$ reported lower NIHSS scores 24 hours after treatment and lower mRS scores 90 days after treatment in SVS+ patients but were not able to confirm the finding in a later study with a larger study population. ${ }^{10}$ Darcourt et $\mathrm{al}^{11}$ reported that SVS+ intracranial vessel occlusions were associated with early neurologic recovery. To our knowledge, no other studies have examined clinical outcome in relation to the SVS after thrombectomy. Our data show superior early neurologic recovery when the SVS was present. Furthermore, SVS+ was associated with better functional outcome and survival 90 days after treatment. Because these findings did not change after factoring in reperfusion success, other factors must have contributed to cause worse outcomes in patients in whom the SVS was absent. We hypothesized that atypical thrombi (septic ${ }^{28}$ or neoplastic $\left.{ }^{29,30}\right)$, which are likely to contain few erythrocytes ${ }^{31}$ and develop after a preceding illness, could be part of the explanation. However, in an additional sensitivity analysis, clinical outcome remained worse for the SVS-group when patients with prestroke dependency ( $\mathrm{mRS}>2$ ) were excluded.

Taking into account that SVS-was also associated with the diagnosis of diabetes mellitus before stroke, further studies on the overall health of patients with SVS-intracranial vessel occlusions are needed to identify underlying, non-stroke-related diseases that may affect thrombus composition ${ }^{32}$ and could explain prestroke dependency, worse outcome, and/or higher mortality rates. According to our data, the occlusion site, for which we adjusted in all our statistical analyses, did not influence reperfusion success or clinical outcome. However, Aoki et $\mathrm{al}^{23}$ have suggested differences in outcome for the proximal-versus-distal M1 SVS. Further studies are needed to determine whether the conclusions drawn about SVS as a potential imaging biomarker depend on the affected vessel segment and/or circulation (ie, anterior versus posterior).

The number of passes performed during MT is a good indicator of the difficulty of clot removal. Although Bourcier et al ${ }^{10}$ provided data on how often $>2$ passes were performed, none of the other studies examining the association between SVS status and reperfusion after MT have addressed this question.,11,12,18 Our data suggest that there is no difference between patients with SVS+ and SVS- in this regard. However, the number of passes performed is at the discretion of the neurointerventionalist in charge and may vary depending on his or her experience and the applicable standards as well as clinical and environmental factors. Although all MTs evaluated in this study were performed by skilled experts, comparability with future studies from different institutions might be limited as a consequence. Further research is necessary to establish reliable guidelines on appropriate MT strategies and the safe number of passes, depending on clinical aspects and imaging characteristics of the occlusive clot.

We hypothesized that intracranial vessel occlusions caused by thromboembolic incidents originating from arteriosclerotic plaques or dissections of the carotid arteries tend to contain a higher number of trapped erythrocytes with deoxygenated hemoglobin (deoxyhemoglobin, intracellular methemoglobin, or hemosiderin) and, thus, are more likely to be SVS+. Our data support that assumption; $96.0 \%$ of patients with severe stenosis or dissection of the carotid arteries had an SVS+ intracranial vessel occlusion. However, we found no association between SVS status and stroke subtypes according to the TOAST classification. Although there are some data indicating that SVS + is associated with a cardioembolic stroke cause, ${ }^{3,9}$ an equal number of studies have found no association between SVS status and stroke subtype whatsoever. ${ }^{8,10}$ Some contraindications to MR imaging (pacemakers, implantable cardioverter-defibrillators, and other metal implants) may have altered the distribution of stroke subtypes in our patient cohort because patients with certain pre-existing medical conditions (eg, heart disease) could have been excluded disproportionately. Advances in the development and increasing use of MR imaging-compatible medical devices could allow the 
inclusion of those patients in future studies. The initial SVS status of intracranial vessel occlusions that resolved without MT may have some diagnostic value with regard to etiology. ${ }^{3,9}$ Further research on how spontaneous reperfusion and the efficiency of IV thrombolysis relate to SVS status is required before the potential of SVS as an imaging biomarker for stroke subtype can be evaluated reliably.

\section{Limitations}

This was a retrospective single-center study, which may limit generalizability. Patients who were not eligible for MR imaging or showed an undeterminable SVS were excluded, possibly causing selection bias. A previous study has shown that baseline criteria and reperfusion outcome of patients with stroke may differ depending on initial imaging technique. ${ }^{33}$ Because most studies examining SVS in patients with AIS used $\mathrm{T}^{*}$ gradient recalledecho sequences, comparability with our study is limited. Although the SVS may give some indication about clot histology, clot composition, which may also impact reperfusion success, was not quantified. The SVS- and SVS+ groups could not be compared with regard to thrombus length and clot burden because the thrombus could only be visualized in patients who showed the SVS. There was no adjustment for occlusions that crossed vessel sections and affected multiple branches (ie, M1-M2), though this aspect might impact first-pass reperfusion and overall reperfusion. Good collateral circulation has been associated with better reperfusion, ${ }^{34}$ but because contralateral angiography was not performed systematically, we were not able to adjust for this factor. We also did not check for early re-occlusion. Future studies could evaluate whether SVS status affects the sustainability of any achieved reperfusion. First-pass expanded TICI, the NIHSS score 24 hours after treatment, and clinical outcome at 90 days could not be assessed for every patient because angiography was not always performed after the first pass and a few patients were lost to follow-up. The resulting data gaps constitute another source of potential selection bias.

\section{CONCLUSIONS}

The SVS is an MR imaging phenomenon frequently observed in patients with AIS, which is associated with successful reperfusion and superior clinical outcome after MT. Our study shows the potential benefits of assessing the SVS in the acute stroke setting. Knowledge of SVS status may influence treatment decisions, improve follow-up care, and refine the assessment of prognosis. Future research will need to assess the diagnostic value of SVS regarding clot composition and patient comorbidities, which may help explain the differences in reperfusion success and clinical outcome observed in this study. However, decisions regarding imaging technique should be made on a case-by-case basis, depending on availability and the patient's clinical condition. Future studies will have to evaluate the overall clinical value of SVS assessment before it can be considered in the diagnostic process.

\section{ACKNOWLEDGMENTS}

We thank Susan Kaplan for editorial assistance.

Disclosures: Marcel Arnold-RELATED: Grant: Swiss National Science Foundation, Swiss Heart Foundation, Comments: research grants*; Consulting Fee or Honorarium:
Bayer HealthCare AG, Bristol Myers Squibb, Covidien, Medtronic, AstraZeneca, Amgen, Daiichi Sankyo, Nestlé Health Science, and Portola, Comments: speaker honoraria for scientific lectures or honoraria for scientific advisory boards. Roland Wiest -RELATED: Grant: Innosuisse grant 43087.1 IP-LS*; UNRELATED: Expert Testimony: Bayer HealthCare AG*; Grants/Grants Pending: Sinergia CRSII5_180365.* Pasquale Mordasini-UNRELATED: Consultancy: Consultant for Medtronic, Cerenovus, MicroVention, Phenox, Siemens*; Grants/Grants Pending: scientific grants from Siemens, iSchemaView*; Payment for Lectures Including Service on Speakers Bureaus: speaker at educational courses for Medtronic.* Jan Gralla—RELATED: Grant: Swiss National Foundation, Comments: Swiss National Science Foundation SNF grant for MRI in stroke*; UNRELATED: Board Membership: Penumbra, Comments: Clinical Event Committee member of the Promise Study (Penumbra), Consultancy*; Consultancy: Medtronic, Comments: Global Principal Investigator of STAR and Swift Direct (Medtronic), Consultancy. * Urs Fischer-UNRELATED: Consultancy: Medtronic, Stryker*; Grants/Grants Pending: Swiss National Science Foundation, Medtronic, Comments: SWIFT DIRECT, BEYOND SWIFT, ELAN, SWITCH.* Johannes Kaesmacher —UNRELATED: Grants/Grants Pending: SAMW/Bangerter Foundation.* *Money paid to institution.

\section{REFERENCES}

1. Flacke S, Urbach H, Keller E, et al. Middle cerebral artery (MCA) susceptibility sign at susceptibility-based perfusion MR imaging: clinical importance and comparison with hyperdense MCA sign at CT. Radiology 2000;215:476-82 CrossRef Medline

2. Schellinger PD, Chalela JA, Kang DW, et al. Diagnostic and prognostic value of early MR imaging vessel signs in hyperacute patients with stroke imaged $<3$ hours and treated with recombinant tissue plasminogen activator. AJNR Am J Neuroradiol 2005;26:618-24 Medline

3. Cho KH, Kim JS, Kwon SU, et al. Significance of susceptibility vessel sign on $\mathrm{T} 2{ }^{\star}$-weighted gradient echo imaging for identification of stroke subtypes. Stroke 2005;36:2379-83 CrossRef Medline

4. Allibert R, Billon Grand C, Vuillier F, et al. Advantages of susceptibility-weighted magnetic resonance sequences in the visualization of intravascular thrombi in acute ischemic stroke. Int $J$ Stroke 2014;9:980-84 CrossRef Medline

5. Kim SK, Yoon W, Kim TS, et al. Histologic analysis of retrieved clots in acute ischemic stroke: correlation with stroke etiology and gradient-echo MRI. AJNR Am J Neuroradiol 2015;36:1756-62 CrossRef Medline

6. Liebeskind DS, Sanossian N, Yong WH, et al. CT and MRI early vessel signs reflect clot composition in acute stroke. Stroke 2011;42:1237-43 CrossRef Medline

7. Powers WJ, Derdeyn CP, Biller J, et al; American Heart Association Stroke Council. 2015 American Heart Association/American Stroke Association Focused Update of the 2013 Guidelines for the Early Management of Patients with Acute Ischemic Stroke Regarding Endovascular Treatment: a Guideline for Healthcare Professionals from the American Heart Association/American Stroke Association. Stroke 2015;46:3020-35 CrossRef Medline

8. Kimura K, Iguchi Y, Shibazaki K, et al. M1 susceptibility vessel sign on $\mathrm{T}^{*}$ as a strong predictor for no early recanalization after IV-tPA in acute ischemic stroke. Stroke 2009;40:3130-32 CrossRef Medline

9. Kang DW, Jeong HG, Kim DY, et al. Prediction of stroke subtype and recanalization using susceptibility vessel sign on susceptibility-weighted magnetic resonance imaging. Stroke 2017;48:1554-59 CrossRef Medline

10. Bourcier R, Mazighi M, Labreuche J, et al; ASTER Trial Investigators. Susceptibility vessel sign in the ASTER trial: Higher recanalization rate and more favourable clinical outcome after first line stent retriever compared to contact aspiration. J Stroke 2018;20:268-76 CrossRef Medline

11. Darcourt J, Withayasuk P, Vukasinovic I, et al. Predictive value of susceptibility vessel sign for arterial recanalization and clinical improvement in ischemic stroke. Stroke 2019;50:512-15 CrossRef Medline 
12. Soize S, Batista AL, Rodriguez Regent C, et al. Susceptibility vessel sign on $\mathrm{T} 2 *$ magnetic resonance imaging and recanalization results of mechanical thrombectomy with stent retrievers: a multicentre cohort study. Eur J Neurol 2015;22:967-72 CrossRef Medline

13. Van Der Marel K, Chueh J-Y, Brooks OW, et al. Quantitative assessment of device-clot interaction for stent retriever thrombectomy. $J$ Neurointerv Surg 2016;8:1278-82 CrossRef Medline

14. Yuki I, Kan I, Vinters HV, et al. The impact of thromboemboli histology on the performance of a mechanical thrombectomy device. AJNR Am J Neuroradiol 2012;33:643-48 CrossRef Medline

15. Dobrocky T, Piechowiak E, Cianfoni A, et al. Thrombectomy of calcified emboli in stroke: Does histology of thrombi influence the effectiveness of thrombectomy? J Neurointerv Surg 2018;10:345-50 CrossRef Medline

16. Duffy S, Mccarthy R, Farrell M, et al. Per-pass analysis of thrombus composition in patients with acute ischemic stroke undergoing mechanical thrombectomy. Stroke 2019;50:1156-63 CrossRef Medline

17. Darcourt J, Garcia C, Phuong DM, et al. Absence of susceptibility vessel sign is associated with aspiration-resistant fibrin/plateletrich thrombi. Int J Stroke 2021 Jan 12. [Epub ahead of print] CrossRef Medline

18. Bourcier R, Volpi S, Guyomarch B, et al. Susceptibility vessel sign on MRI predicts favorable clinical outcome in patients with anterior circulation acute stroke treated with mechanical thrombectomy. AJNR Am J Neuroradiol 2015;36:2346-53 CrossRef Medline

19. Liu M, Li L, Li G. The different clinical value of susceptibility vessel sign in acute ischemic patients with stroke under different interventional therapy: a systematic review and meta-analysis. J Clin Neurosci 2019;62:72-79 CrossRef Medline

20. Adams HP, Bendixen BH, Kappelle LJ, et al. Classification of subtype of acute ischemic stroke: definitions for use in a multicenter clinical trial-TOAST. Trial of Org 10172 in Acute Stroke Treatment. Stroke 1993;24:35-41 CrossRef Medline

21. Liebeskind DS, Bracard S, Guillemin F, et al; HERMES Collaborators. ETICI reperfusion: defining success in endovascular stroke therapy. J Neurointerv Surg 2019;11:433-38 CrossRef Medline

22. Hacke W, Kaste M, Fieschi C, et al. Randomised double-blind placebo-controlled trial of thrombolytic therapy with intravenous alteplase in acute ischaemic stroke (ECASS II): Second European-Australasian Acute Stroke Study Investigators. Lancet 1998;352:1245-51 CrossRef Medline
23. Lapergue B, Blanc R, Gory B, et al; ASTER Trial Investigators. Effect of endovascular contact aspiration vs stent retriever on revascularization in patients with acute ischemic stroke and large vessel occlusion: the ASTER randomized clinical trial. JAMA 2017;318:443-52 CrossRef Medline

24. Aoki J, Kimura K, Shibazaki K, et al. Location of the susceptibility vessel sign on $\mathrm{T} 2{ }^{*}$-weighted MRI and early recanalization within 1 hour after tissue plasminogen activator administration. Cerebrovasc Dis Extra 2013;3:111-20 CrossRef Medline

25. Kimura K, Sakamoto Y, Aoki J, et al. Clinical and MRI predictors of no early recanalization within 1 hour after tissue-type plasminogen activator administration. Stroke 2011;42:3150-55 CrossRef Medline

26. Hashimoto T, Hayakawa M, Funatsu N, et al. Histopathologic analysis of retrieved thrombi associated with successful reperfusion after acute stroke thrombectomy. Stroke 2016;47:3035-37 CrossRef Medline

27. Gunning GM, Mcardle K, Mirza M, et al. Clot friction variation with fibrin content; implications for resistance to thrombectomy. $J$ Neurointerv Surg 2018;10:34-38 CrossRef Medline

28. Ruttmann E, Willeit J, Ulmer H, et al. Neurological outcome of septic cardioembolic stroke after infective endocarditis. Stroke 2006;37:2094-99 CrossRef Medline

29. Kneihsl M, Enzinger C, Wünsch G, et al. Poor short-term outcome in patients with ischaemic stroke and active cancer. J Neurol 2016;263:150-56 CrossRef Medline

30. Aarnio K, Joensuu H, Haapaniemi E, et al. Cancer in young adults with ischemic stroke. Stroke 2015;46:1601-06 CrossRef Medline

31. Fu $\mathrm{CH}$, Chen $\mathrm{CH}$, Lin $\mathrm{YH}$, et al. Fibrin and platelet-rich composition in retrieved thrombi hallmarks stroke with active cancer. Stroke 2020;51:3723-27 CrossRef Medline

32. Ye G, Gao Q, Qi P, et al. The role of diabetes mellitus on the thrombus composition in patients with acute ischemic stroke. Interv Neuroradiol 2020;26:329-36 CrossRef Medline

33. Meinel TR, Kaesmacher J, Mosimann PJ, et al. Association of initial imaging modality and futile recanalization after thrombectomy. Neurology 2020;95:e2331-42 CrossRef Medline

34. Bang OY, Saver JL, Kim SJ, et al. Collateral flow predicts response to endovascular therapy for acute ischemic stroke. Stroke 2011;42:69399 CrossRef Medline 